\section{Two new Asterina species on Michelia champaca from Kerala, India}

\author{
V.B. Hosagoudar ${ }^{1} \&$ M.C. Riju ${ }^{2}$ \\ 1,2 Tropical Botanic Garden and Research Institute, Palode, \\ Thiruvananthapuram, Kerala 695562, India. \\ Email: ${ }^{1}$ vbhosagoudar@rediffmail.com (corresponding author), \\ 2rcmakkiyil@gmail.com
}

The genus Michelia comprises 50 species, of which four are in India. Michelia champaca L. and $M$. nilagirica Zenk are known from Kerala State (Santapau \& Henry 1984; Sasidharan 2004; Nayar et al. 2006). The former species is endemic to South and South East Asia, while the latter is endemic to the southern Western Ghats and Sri Lanka. Hansford (1947) and Hosagoudar \& Goos (1996) have described Asterina micheliae and Asterostomella micheliae on the latter host from Sri Lanka and the southern Western Ghats (Idukki forest region) of peninsular India, respectively. Our recent collections of Michelia champaca from the Wyanad region of Kerala State revealed two undescribed species of the genus Asterina and they are described and illustrated here in detail. Key to the Asterina species known on the host genus Michelia has been provided.

Date of publication (online): 26 July 2011

Date of publication (print): 26 July 2011

ISSN $0974-7907$ (online) | 0974-7893 (print)

Editor: R.K. Verma

Manuscript details:

Ms \# 02746

Received 05 April 2011

Final received 26 May 2011

Finally accepted 06 June 2011

Citation: Hosagoudar, V.B. \& M.C. Riju (2011). Two new Asterina species on Michelia champaca from Kerala, India. Journal of Threatened Taxa 3(7): 1942-1946.

Copyright: (C) V.B. Hosagoudar \& M.C. Riju 2011. Creative Commons Attribution 3.0 Unported License. JoTT allows unrestricted use of this article in any medium for non-profit purposes, reproduction and distribution by providing adequate credit to the authors and the source of publication.

Acknowledgements: We thank Dr. A. Subramoniam, Director, TBGRI Palode for the facilities.

\section{OPEN ACCESS | FREE DOWNLOAD}

Asterina michelifolia sp. nov. (Images 1-5, Fig. 1)

\section{Materials examined:}

20.ix.2008, on leaves of Michelia champaca L. (Magnoliaceae), Chennalode, Padinharathara, Wyanad, Kerala, India, coll. M.C. Riju, HCIO 49111a (holotype), TBGT 3366a (isotype). MycoBank No. (MB 561622).

Coloniae hypophyllae, tenues, ad $2 \mathrm{~mm}$ diam., confluentes. Hyphae flexuosae vel anfractuae, irregulariter acuteque vel laxe ramosae, formans retes arte reticulatae, cellulae $12-40$ × 3-5 $\mu \mathrm{m}$. Appressoria dispersa, unicellularis, opposita, alternata, unilateralis, antrorsa vel retrorsa, globosa vel cylindracea, integra, $5-18 \times 5-8 \mu \mathrm{m}$. Pycnothyria dispersa, orbicularis, ad $58 \mu \mathrm{m}$ diam., stellatim dehiscentes et perlate orificium ad centro; pycnothyriosporae globosae, clavatae, 15-20 $\mu \mathrm{m}$, parietus glabrus. Thyriothecia dispersa, orbicularis, ad $85 \mu \mathrm{m}$ diam., stellatim dehiscentes et perlate orificium ad centro et asci distinctum; asci globosi vel ovati, $37-45 \mu \mathrm{m}$ diam.; ascosporae brunnneae, uniseptatae, constrictus ad septatum, 22$25 \times 10-13 \mu \mathrm{m}$, parietus glabrus.

Colonies hypophyllous, thin, up to $2 \mathrm{~mm}$ in diameter, confluent. Hyphae flexuous to crooked, branching irregular at acute to wide angles, forming closely reticulated rings, cells $12-40 \times 3-5 \mu \mathrm{m}$. Appressoria scattered, unicellular, opposite, alternate, unilateral, antrorse to retrorse, globose to cylindrical, entire, 5-18 x 5-8 $\mu \mathrm{m}$. Pycnothyria scattered, orbicular, up to $58 \mu \mathrm{m}$ in diameter, stellately dehisced and widely opened at the centre; pycnothyriospores globose, clavate, 15-20 $\mu \mathrm{m}$, wall smooth. Thyriothecia scattered, orbicular, up to $85 \mu \mathrm{m}$ in diameter, stellately dehisced and widely opened at the centre by exposing asci; asci globose to ovate, $37-45 \mu \mathrm{m}$ in diameter; ascospores brown, uniseptate, constricted at the septum, 22-25 x 10-13 $\mu \mathrm{m}$, wall smooth.

Etymology: Specific epithet based on the host genus.

This species differs from Asterina micheliae Hansf. reported on Michelia nilagirica from Sri Lanka (Hansford 1947) in having typical thyriothecium and differs from $A$. micheligena in having straight mycelium and larger ascospores. 


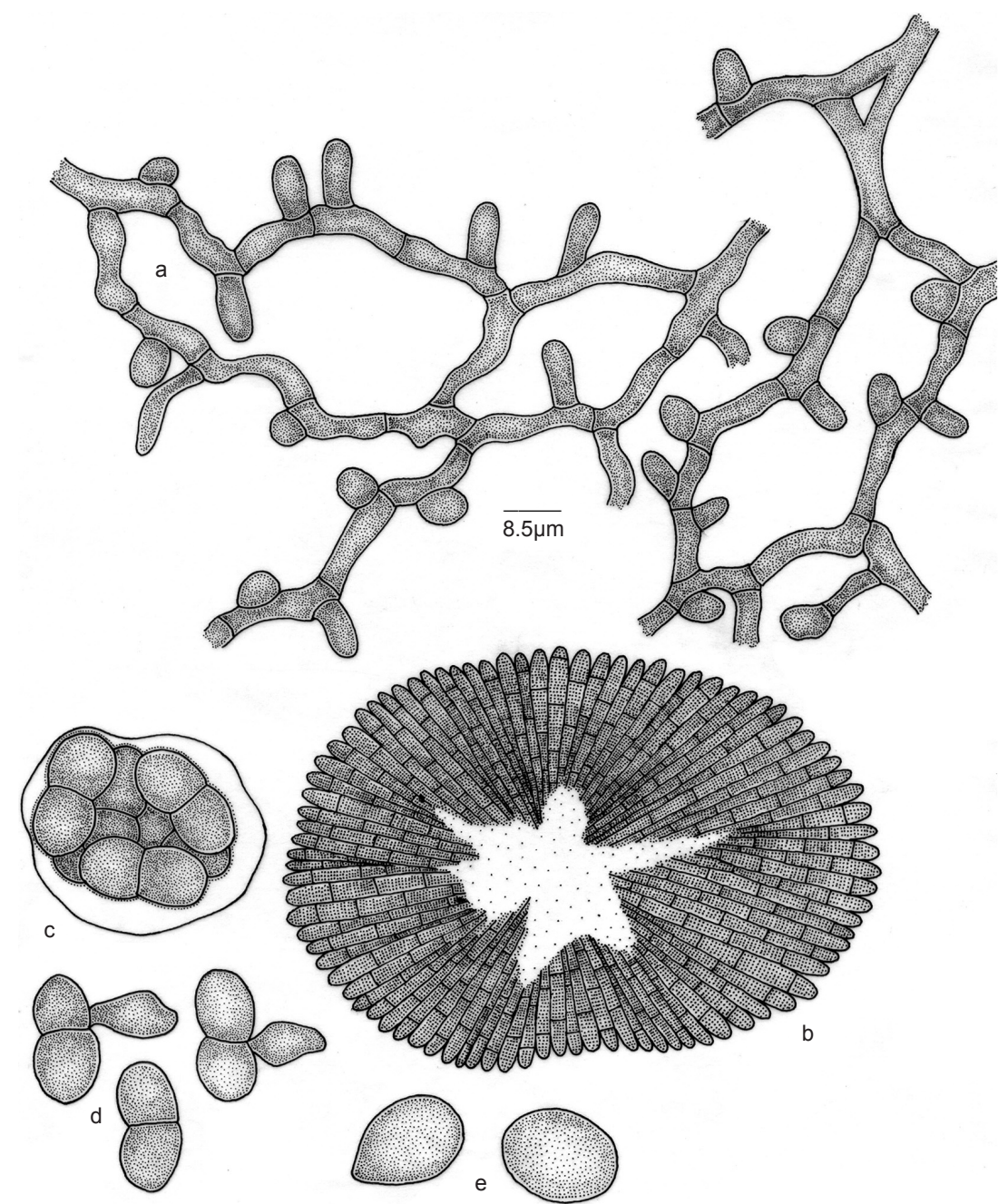

Figure 1. Asterina michelifolia sp. nov. a - apprassoriate mycelium; b - thyriothecium;

c - ascus; d - ascospores;

e - pycnothyriospores
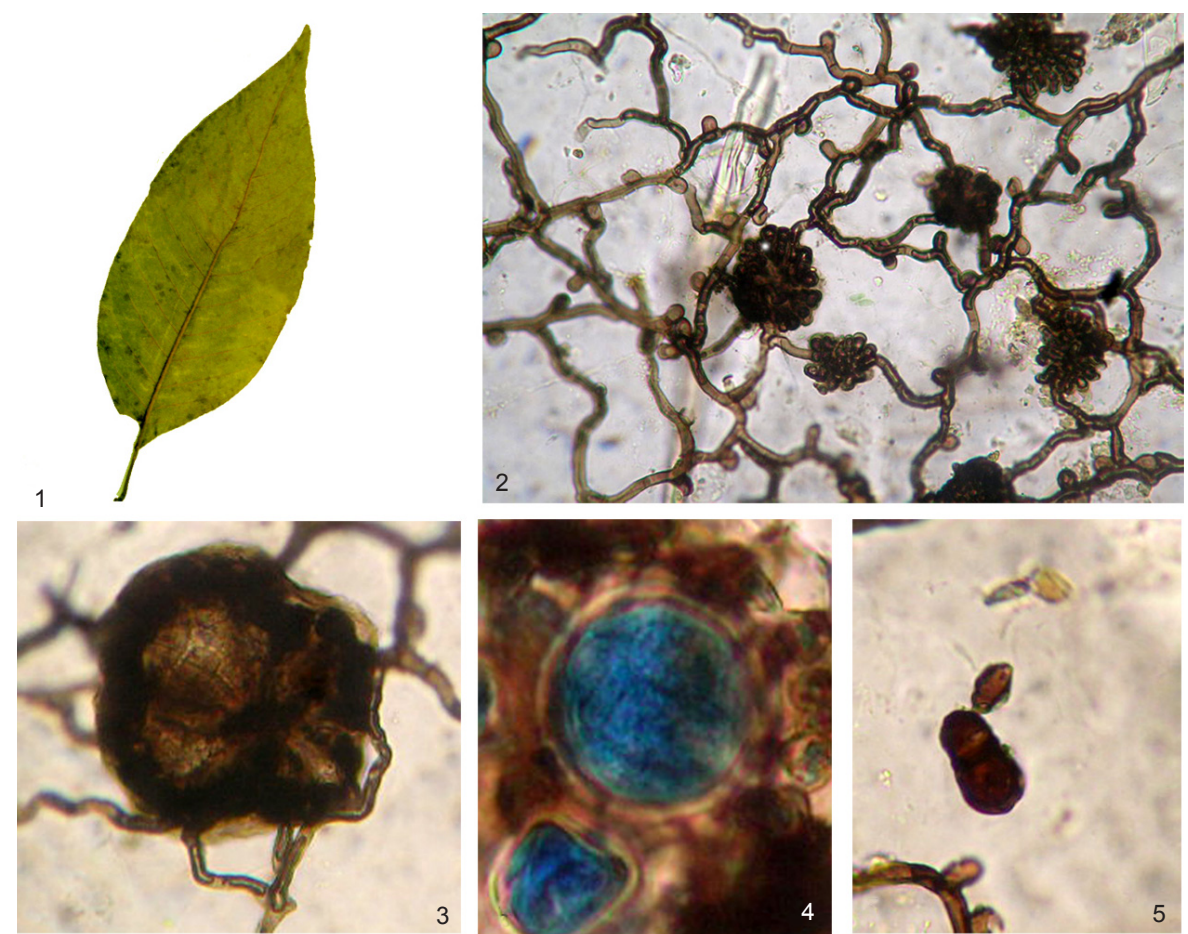

Images 1-5. Asterina michelifolia sp. nov.

1 - infected leaf;

2 - colony with thyriothecia;

3 - thyriothecium;

4 - asci;

5 - germinating ascospore

Journal of Threatened Taxa | www.threatenedtaxa.org | July 2011 | 3(7): 1942-1946 
Asterina micheligena sp. nov. (Images 6-11, Fig. 2)

Materials examined: 20.ix.2008, on leaves of Michelia champaca L. (Magnoliaceae), Chennalode, Padinharathara, Wyanad, Kerala, India, coll. M.C. Riju HCIO 49111b (holotype), TBGT 3366b (isotype). MycoBank No. (MB 561623).

Coloniae epiphyllae, densae, ad $3 \mathrm{~mm}$ diam., confluentes et saepe et nervicolae. Hyphae flexuosae vel anfractuae, irregulariter acuteque vel laxe ramosae, formans retes arte reticulatae, cellulae $12-40 \times 3-5 \mu \mathrm{m}$. Appressoria dispersa, unicellularis, opposita, alternata, unilateralis, antrorsa vel retrorsa, globosa, integra, mammiformes, 4-7 x 4-9 $\mu \mathrm{m}$. Pycnothyria dispersa, orbicularis, ad $75 \mu \mathrm{m}$ diam., stellatim dehiscentes et perlate orificium ad centro; pycnothyriosporae globosae vel leniter ovatae, $17-25 \mu \mathrm{m}$ diam., parietus glabrus. Thyriothecia dispersa, orbicularis, ad $188 \mu \mathrm{m}$ diam., stellatim dehiscentes et perlate orificium ad centro et asci distinctum; asci globosi vel ovati, ad $63 \mu \mathrm{m}$ diam.; ascosporae brunneae, uniseptatae, constrictus ad septatum, 25-33 x15-18 $\mu \mathrm{m}$, parietus glabrus.

Colonies epiphyllous, dense, up to $3 \mathrm{~mm}$ in diameter, confluent and often trait along the major veins of the upper surface of the leaves. Hyphae substraight to flexuous, branching opposite, alternate to irregular at acute to wide angles, loosely to closely reticulate, cells 9-24 x 4-6 $\mu \mathrm{m}$. Appressoria scattered, unicellular, opposite, alternate, unilateral, globose, entire, mammiform, 4-7 x 4-9 $\mu \mathrm{m}$. Pycnothyria scattered, orbicular, up to $75 \mu \mathrm{m}$ in diameter, stellately dehisced and widely opened at the centre; pycnothyriospores
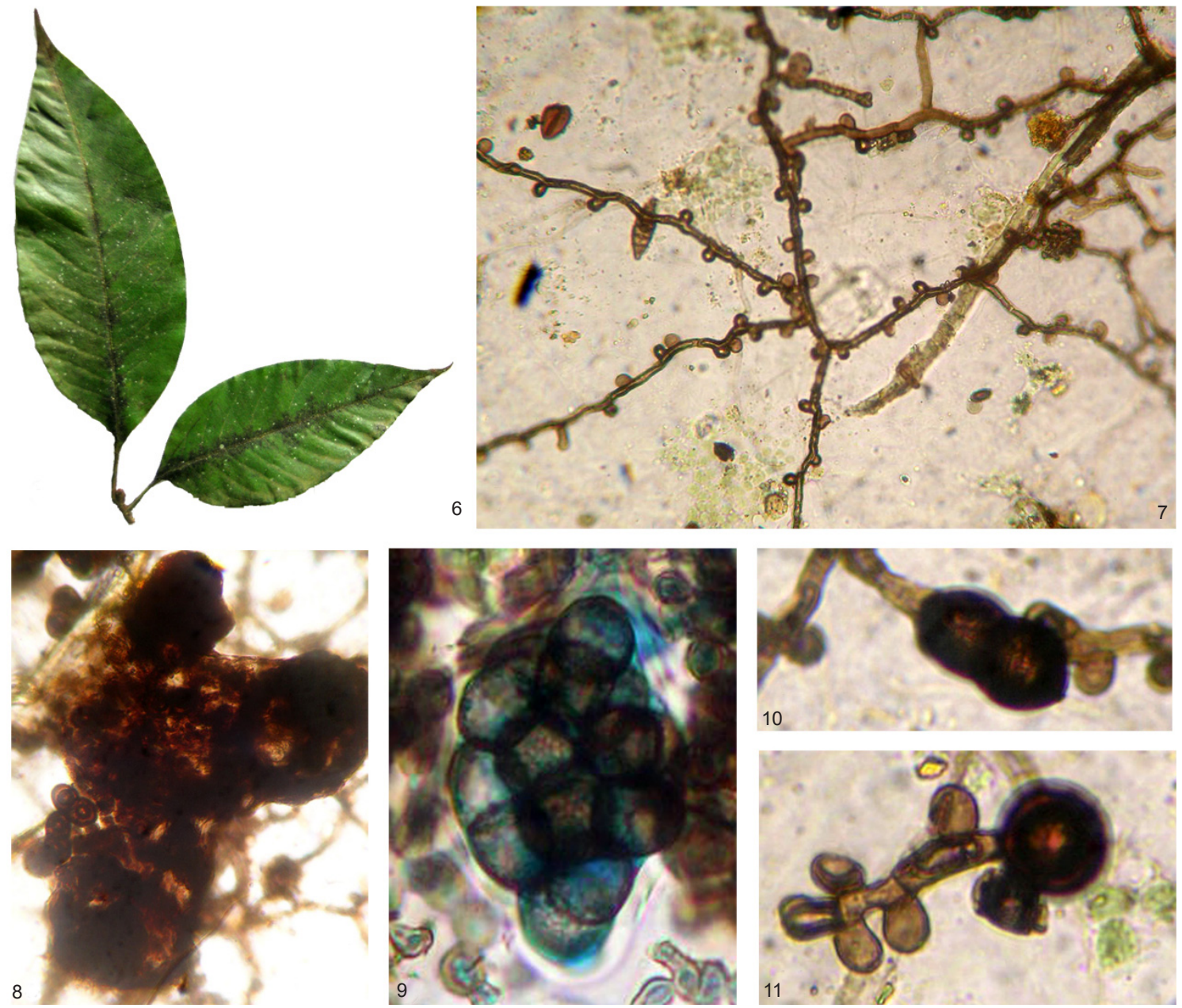

Images 6-11. Asterina micheligena sp. nov.

6 - infected leaves; 7 - colony with apressoriate mycelium; 8 - thyriotheca; 9 - asci; 10 - germinating ascospore;

11 - germinating pycnothyriospore 


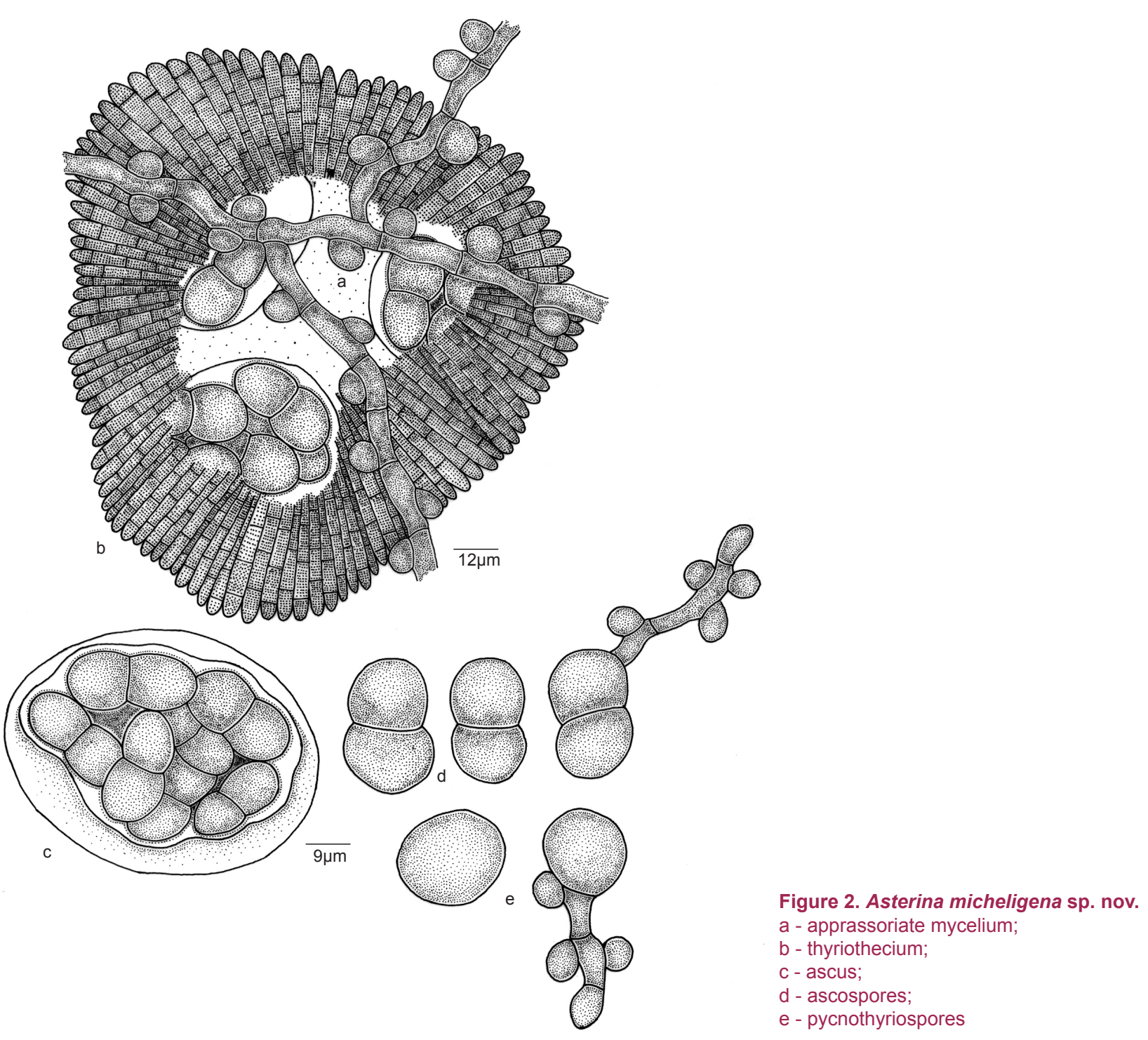

Key to the Asterina species on the genus Michelia

Only anamorph known Asteostomella micheliae Teleomorph known

Asterina micheliae

Mature fruiting body resembles perithecia ........................ 3 Mature fruiting body resembles thyriothecia Asterina michelifolia sp. nov.

less than $28 \mu \mathrm{m}$ long

.

Ascospores more than $15 \mu \mathrm{m}$ broad .................................................... Asterina micheligena sp. nov. Ascospores less than $15 \mu \mathrm{m}$ broad.......

A. manglietiae

globose to slightly ovate, $17-25 \mu \mathrm{m}$ in diameter, wall smooth. Thyriothecia scattered, orbicular, up to $188 \mu \mathrm{m}$ in diameter, stellately dehisced and widely opened at the centre by exposing asci; asci globose to ovate, up to $63 \mu \mathrm{m}$ in diameter; ascospores brown, uniseptate, constricted at the septum, $25-33 \times 15-18 \mu \mathrm{m}$, wall smooth.

Etymology: Specific epithet based on the host genus. 


\section{REFERENCES}

Hosagoudar, V.B. \& R.D. Goos (1996). Some foliicolous fungi from southern India. Mycotaxon 59: 149-166.

Hansford, G.C. (1947). New or interesting tropical fungi I. Proceedings of the Linnaean Society of London 158: 28-50.

Nayar, T.S.,A.R. Beegam, N. Mohanan \& G.R. Kumar(2006). Flowering Plants of Kerala. Tropical Botanic Garden and Research Institute, Palode, Thiruvananthapuram, Kerala, India, 1069pp.
Santapau, H. \& A.N. Henry (1984). A Dictionary of Flowering Plants in India. CSIR New Delhi, 198pp.

Sasidharan, N. (2004). Biodiversity Documentation for Kerala - Part 6: Flowering Plants. Kerala Forest Research Institute, Peechi, Kerala, 437pp.

Song, B., T.H. Li \& Y.H. Shen (2001). Two new taxa of Asterina on Magnoliaceae from China. Mycosystema 20(4): 461-463. 